\title{
Doping Induced Tailoring in the Morphology, Band-Gap and Ferromagnetic Properties of Biocompatible ZnO Nanowires, Nanorods and Nanoparticles
}

\author{
Javed Iqbal ${ }^{1,2, *}$, Tariq $\mathrm{Jan}^{2}$, Yu Ronghai ${ }^{1,3}$, Sajjad Haider Naqvi ${ }^{4}, \quad$ Ishaq Ahmad $^{5}$
}

(Received 14 December 2013; accepted 04 April 2014; published online July 1, 2014)

\begin{abstract}
The modification of nanostructured materials is of great interest due to controllable and unusual inherent properties in such materials. Single phase Fe doped ZnO nanostructures have been fabricated through simple, versatile and quick low temperature solution route with reproducible results. The amount of Fe dopant is found to play a significant role for the growth of crystal dimension. The effect of changes in the morphology can be obviously observed in the structural and micro-structural investigations, which may be due to a driving force induced by dipole-dipole interaction. The band gap of $\mathrm{ZnO}$ nanostructures is highly shifted towards the visible range with increase of Fe contents, while ferromagnetic properties have been significantly improved. The prepared nanostructures have been found to be nontoxic to SH-SY5Y Cells. The present study clearly indicates that the Fe doping provides an effective way of tailoring the crystal dimension, optical band-gap and ferromagnetic properties of $\mathrm{ZnO}$ nanostructure-materials with nontoxic nature, which make them potential for visible light activated photocatalyst to overcome environmental pollution, fabricate spintronics devices and biosafe drug delivery agent.
\end{abstract}

Keywords: ZnO; Fe doping; Dipole-dipole interaction; Band-gap tailoring; Ferromagnetism; Cytotoxicity

Citation: Javed Iqbal, Tariq Jan, Yu Ronghai, Sajjad Haider Naqvi and Ishaq Ahmad, "Doping Induced Tailoring in the Morphology, Band-Gap and Ferromagnetic Properties of Biocompatible ZnO Nanowires, Nanorods and Nanoparticles", Nano-Micro Lett. 6(3), 242-251 (2014). http://dx.doi.org/10.5101/nml140026a

\section{Introduction}

$\mathrm{ZnO}$ is an important group of II-VI semiconductor with the characteristics of wide direct band gap (3.37 $\mathrm{eV})$, large exciton binding energy $(60 \mathrm{meV})$, inexpensive and environmentally safe material $[1,2]$. Due to these remarkable characteristics, $\mathrm{ZnO}$ nanomaterials have attracted a lot of research interest in the field of science and technology [3,4]. Among $\mathrm{ZnO}$ nanomaterials family, the one dimensional (1D) $\mathrm{ZnO}$ nanostructures (such as nanowires, nanorods, nanofibers and nanotubes etc) are of special interest because of their high surface area and compatibility with the functional nanodevices. Due to these significant properties, the $1 \mathrm{D} \mathrm{ZnO}$ nanostructures has found large number of applications in many devices such as nano lasers, single nanowire field effect transistor, UV blockers and detectors, power generators and gas sensors [5-9]. For all these devices, the controlled nanoscale morphological characteristics are highly desired.

\footnotetext{
${ }^{1}$ Laboratory of Advanced Materials, Department of Material Science and Engineering, Tsinghua University, Beijing, China

${ }^{2}$ Laboratory of Nanoscience and Technology, Department of Physics, International Islamic University Islamabad, Pakistan

${ }^{3}$ School of Materials Science and Engineering, Beihang University, Beijing, China

${ }^{4}$ Karachi University, Karachi, Pakistan

${ }^{5}$ Nanational Center for Physics (NCP), Quaid-i-Azam University, Islamabad, Pakistan

*Corresponding author. javed.saggu@iiu.edu.pk
} 
In these days, $\mathrm{ZnO}$ based photocatalyst is considered to be an excellent substitute of $\mathrm{TiO}_{2}$ to overcome the current global environmental pollution problem due to its good stability and non-toxic nature [10]. However, the large band gap of $\mathrm{ZnO}$ lies in the UV range, which allows for only $\sim 6 \%$ of sunlight to be useful for the activation of the catalyst. Therefore, a visible light activated catalyst is highly desired that can take advantage of a larger fraction of the solar spectrum and would be much more effective in environmental cleanup [11]. It has been found that the optical band gap energy of $\mathrm{ZnO}$ nanostructures can be effectively tailored via doping of different transitional metals (TMs) [12]. In addition, currently a lot of experimental and theoretical research is also concentrated on $\mathrm{TM}$ doped $\mathrm{ZnO}$ dilute magnetic semiconductors (DMSs) because of their room temperature ferromagnetic behavior [13]. ZnO nanostructures with tailored optical and magnetic properties may have useful applications in future spintronics devices [13]. In order to look above functionality with morphological modification, optical improvements and room temperature ferromagnetism with high $T_{c}$, Fe is supposed to be a potential candidate for doping in $\mathrm{ZnO}$.

In addition, the inorganic nanostructures have an important role in biomedical sector because of their significant photocatalytic and antibacterial characteristics [14]. Among these, $\mathrm{ZnO}$ nanostructures are of special attention because of their cost effectiveness and established applications in health care products [15]. $\mathrm{ZnO}$ could also be used for drug delivery but toxicity concern is there. Bulk $\mathrm{ZnO}$ is considered as safe material by FDA (US Food and Drug Administration) but when reduced to the nanoscale, $\mathrm{ZnO}$ can develop new actions of toxicity [16].

In this work, Fe doped $\mathrm{ZnO}$ nanostructures including nanoparticles, nanorods and nanowires based on the soft chemistry synthetic approach have been prepared at room temperature. A series of Fe doped $\mathrm{ZnO}$ samples with varying $\mathrm{Fe}$ contents have been systematically studied. The band gap of $\mathrm{ZnO}$ has been dramatically reduced with the increase in Fe doping level. The morphology of doped samples shows an interesting change from $1 \mathrm{D}$ to zero dimension (0D). The resulting Fe doped $\mathrm{ZnO}$ nanostructures possess both tuned optical and magnetic properties, which will enable them for designed industrial applications. Furthermore, the cytotoxicity of $\mathrm{Fe}$ doped $\mathrm{ZnO}$ nanostructures has been studied and found to be nontoxic for SH-SY5Y Cells.

\section{Experimental}

Fe doped $\mathrm{ZnO}$ nanostructures have been prepared using wet chemical method as in our previous reported work $[17,18]$. Different analytical grade chemicals have been used for the fabrication of ultrafine nanostruc- tures. In a typical synthesis process, $0.5 \mathrm{M} \mathrm{NaOH}$ solution prepared in ethanol as precipitating agent has been added drop by drop into molar solution of $0.05 \mathrm{M}$ $\mathrm{Zn}\left(\mathrm{NO}_{3}\right)_{2} \cdot 6 \mathrm{H}_{2} \mathrm{O}$ with different stoichiometric ratio of $\mathrm{FeCl}_{3} \cdot 6 \mathrm{H}_{2} \mathrm{O}$ at normal laboratory temperature for $1 \mathrm{~h}$.

$$
\begin{aligned}
& \mathrm{Zn}\left(\mathrm{NO}_{3}\right)_{2}+\mathrm{NaOH} \rightarrow \mathrm{Zn}\left(\mathrm{NO}_{3}\right)(\mathrm{OH})+\mathrm{NaNO}_{3} \\
& \mathrm{Zn}\left(\mathrm{NO}_{3}\right)(\mathrm{OH})+\mathrm{NaOH} \rightarrow \mathrm{ZnO}+\mathrm{NaNO}_{3}+\mathrm{H}_{2} \mathrm{O}
\end{aligned}
$$

After one hour of vigorous magnetic stirring, the solution has been put into an Autoclave (Teflon vessel) and heated at $120^{\circ} \mathrm{C}$ for $15 \mathrm{~h}$. The precipitates have been collected, washed and then centrifuged. Finally, these washed precipitates have been dried in oven at $110^{\circ} \mathrm{C}$. Different characterization techniques have been employed as were used in our previous reported work $[17,18]$.

The SH-SY5Y cells of human being have been used as target which are commercial available at ATTC, USA. The cells have been cultured at $37^{\circ} \mathrm{C}$ in DMEM medium for $48 \mathrm{~h}$ with in humidified environment. The colloidal solution of the synthesized nanostructures with different $\mathrm{Fe}$ doping level has been used against these cells. The cells cultured without the presence of these nanostructures have been used as control for comparison.

\section{Results and discussion}

\section{Morphology and microstructure}

The morphology and microstructure of these prepared crystalline samples have been carefully investigated using electron microscopy techniques. Figure 1(a), (b), (c) and (d) demonstrate the TEM, HRTEM and SAED images of Fe doped $\mathrm{ZnO}$ with different compositions $\mathrm{x}=0.5 \%, 1 \%, 2 \%$ and $5 \%$ of Fe dopant, respectively. It has been interestingly found that the prepared doped samples undergo a significant morphological changes induced by the amount of Fe dopant concentrations into $\mathrm{ZnO}$ structure. The pure $\mathrm{ZnO}$ sample portrays the nanowires of length $5 \mu \mathrm{m}$ and their growth direction is preferably oriented along c-axis. Figure 1 (a) shows the morphology of $\mathrm{Fe}_{0.5} \mathrm{Zn}_{99.5} \mathrm{O}$ sample, which exhibits nanowires of average length $3 \mu \mathrm{m}$ with $40 \mathrm{~nm}$ of diameter. The microstructure study with HRTEM of grown nanowires shows single phase structure with the interspacing between planes of $0.22 \mathrm{~nm}$ and length of caxis $0.5204 \mathrm{~nm}$, which reveals that the growth direction of these nanowires is preferably oriented along (0001) as shown in Fig. 1(a). The sample with the nominal composition of $\mathrm{x}=1 \%$ has a morphology of nanorods within the average length of $900 \mathrm{~nm}$ and a diameter of $90 \mathrm{~nm}$ as shown in Fig. 1(b). The microstructural HRTEM study of these as prepared nanorods shows that the c-axis lattice constant value is little reduced and the spacing between planes is also changed. 

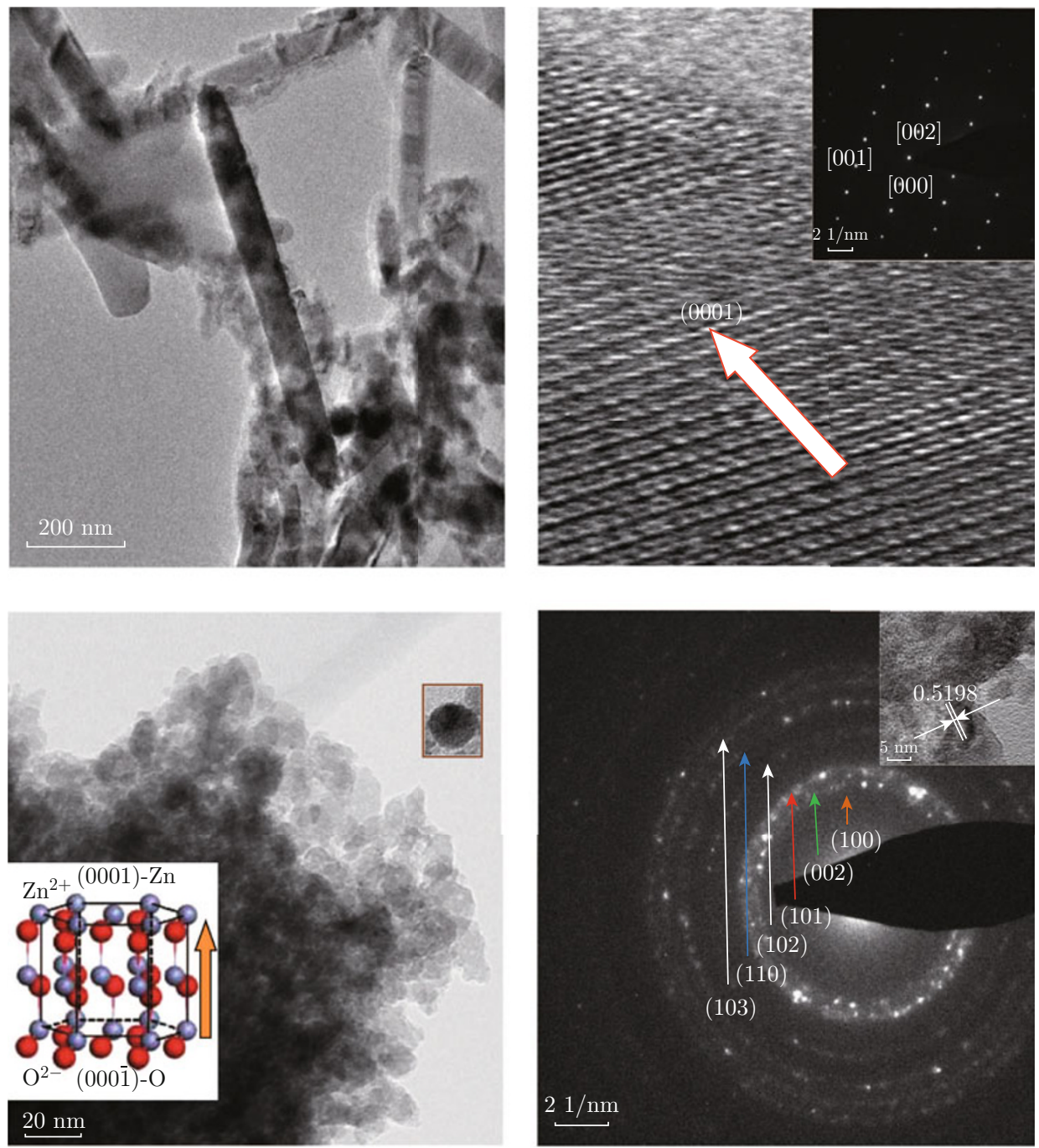

Fig. 1 TEM, HRTEM and SAED patterns of Fe $(\mathrm{x}=0.5 \%, 1 \%, 2 \%$ and $5 \%)$ doped ZnO Nanostructures.

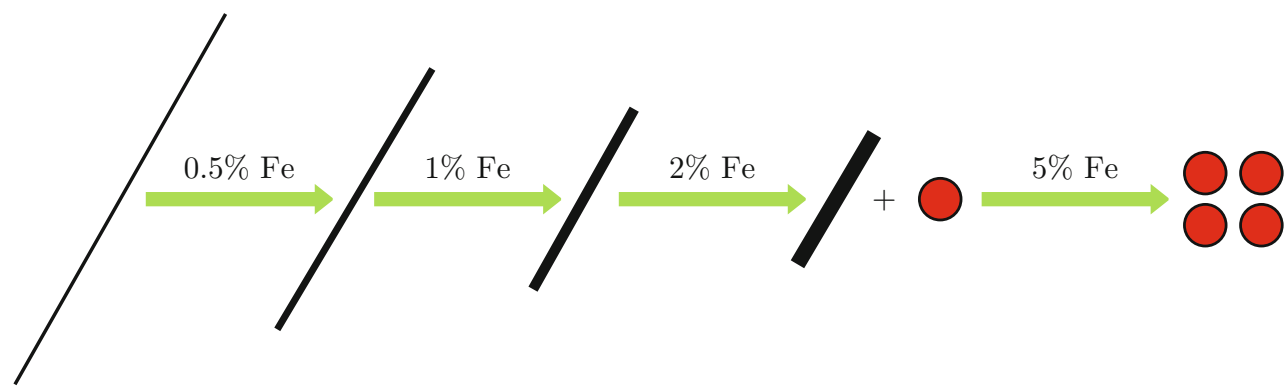

Diagram 1 Schematic drawing of Fe doping induced tailoring in the morphology

However, the absence of secondary phases can be ruled out from Fig. 1(b). With the increase of Fe doping concentration up to $2 \%$, there are two kinds of mixed morphology consisting of nanorods and nanoparticles as shown in Fig. 1(c). The major part of morphology is nanorods, whose micro-structural investigation in high resolution shows that the growth along c-axis planes is highly influenced and the micro-structure has mixed morphology overlook. In order to confirm the structure formation, SAED has been carried out for this sample and is depicted in the Fig. 1(c). All resolved fringes pattern of diffraction shows the single phase wurtzite structure formation. When the concentration of Fe dopant is increased up to $5 \%$, the morphology is dramatically changed from 1D to nanoparticles as shown in Fig. 1(d). The average size of these nanoparticles lies in the range of $12 \mathrm{~nm}$. The HRTEM and SAED investigations of these prepared nanoparticles demon- 
strate that they are in $\mathrm{ZnO}$ wurtzite single phase and all selected area diffraction fringes can be indexed to hexagonal wurtzite structure as shown in Fig. 1(d). In order to make the picture of morphology change more clear, it has been noted that with the introduction of Fe into $\mathrm{ZnO}$ lattice, the growth along c-axis is affected and the length of nanowires and nanorods is decreased, while diameter increased, which gives the evidences of some force to hinder the growth of crystal along $c$-axis. There is overall a schematic change in the morphology with the variation of $\mathrm{Fe}$ doping concentration as depicted in diagram 1.

The growth mechanism of nanocrystal structure can be explained on the basis of the polar surfaces of $\mathrm{ZnO}$. The hexagonal wurtzite structure of $\mathrm{ZnO}$ has a series of a number of alternating planes which are made of tetrahedral coordinated $\mathrm{O}^{2-}$ and $\mathrm{Zn}^{2+}$ ions, piling up along the $c$-axis [19]. The ions create positively charged (0001)-Zn and negatively charged (000-1)-O polar surfaces as shown in the inset of Fig. 1(d) [20]. The incor-
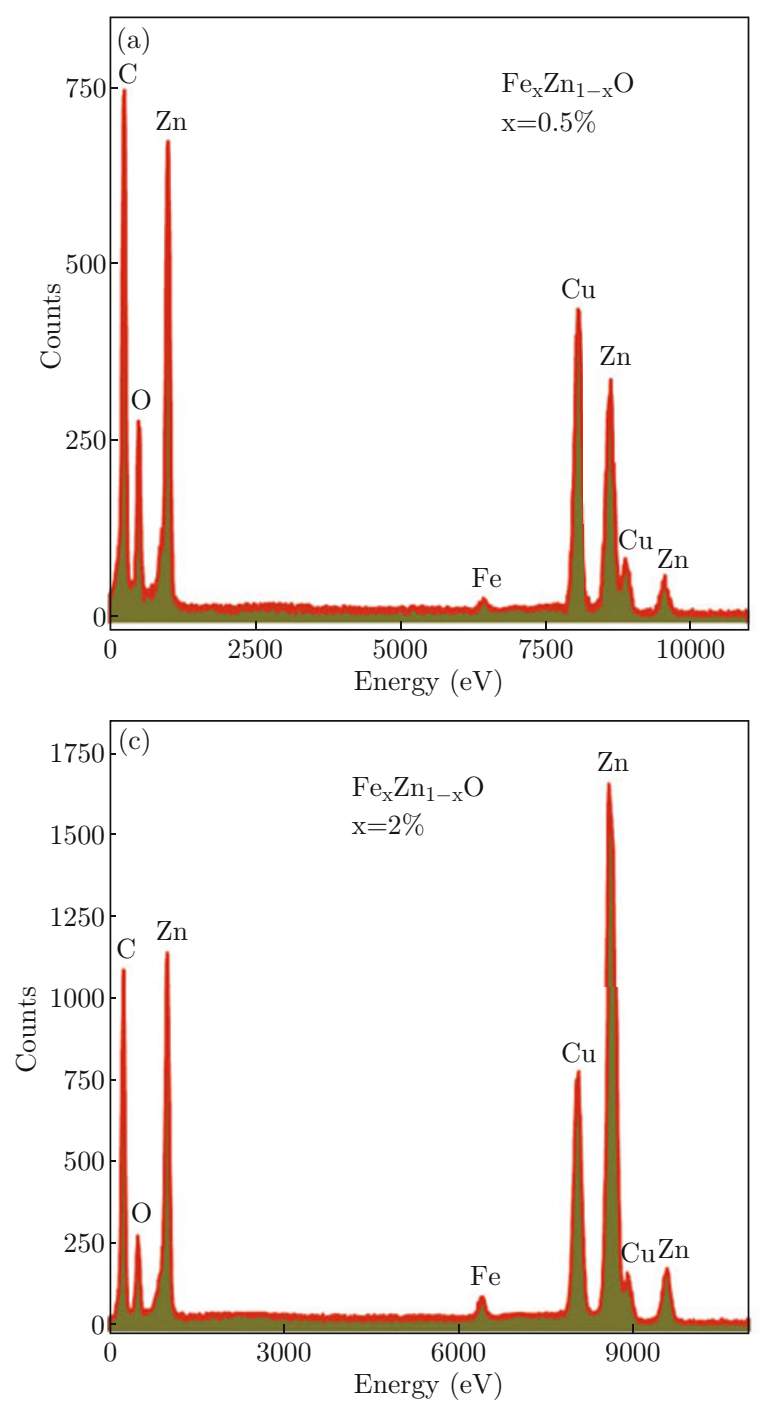

poration of $\mathrm{Fe}^{3+}$ into $\mathrm{ZnO}$ matrix can produce planar defects along (0001) plane which may reduce the surface energy and result in the quick anisotropic growth along different direction instead of c-axis without affecting the intrinsic polarity of nanostructures. The change of morphology from nanowires to nanorods and then nanoparticles may be due to the reason that polar surfaces tend to minimize their surface area. The electrostatic energy may be reduced by interfacing of (0001)-Zn plane with (0001)-O plane. This interfacing will balance the local polar charges and leads to reduce surface area. In brief, the change in the morphology as a function of doping concentration may be due to the increase of charge carriers of $\mathrm{Fe}^{3+}$ to replace $\mathrm{Zn}^{2+}$ sites into lattice, the positive charge on c-axis growth plane increases and there is driving force produced by dipole-dipole electrostatics interaction, which hinders the growth along one direction [21].

Figure 2 demonstrates the EDS spectra of Fe doped $\mathrm{ZnO}$ nanostructures. The spectra of all samples show
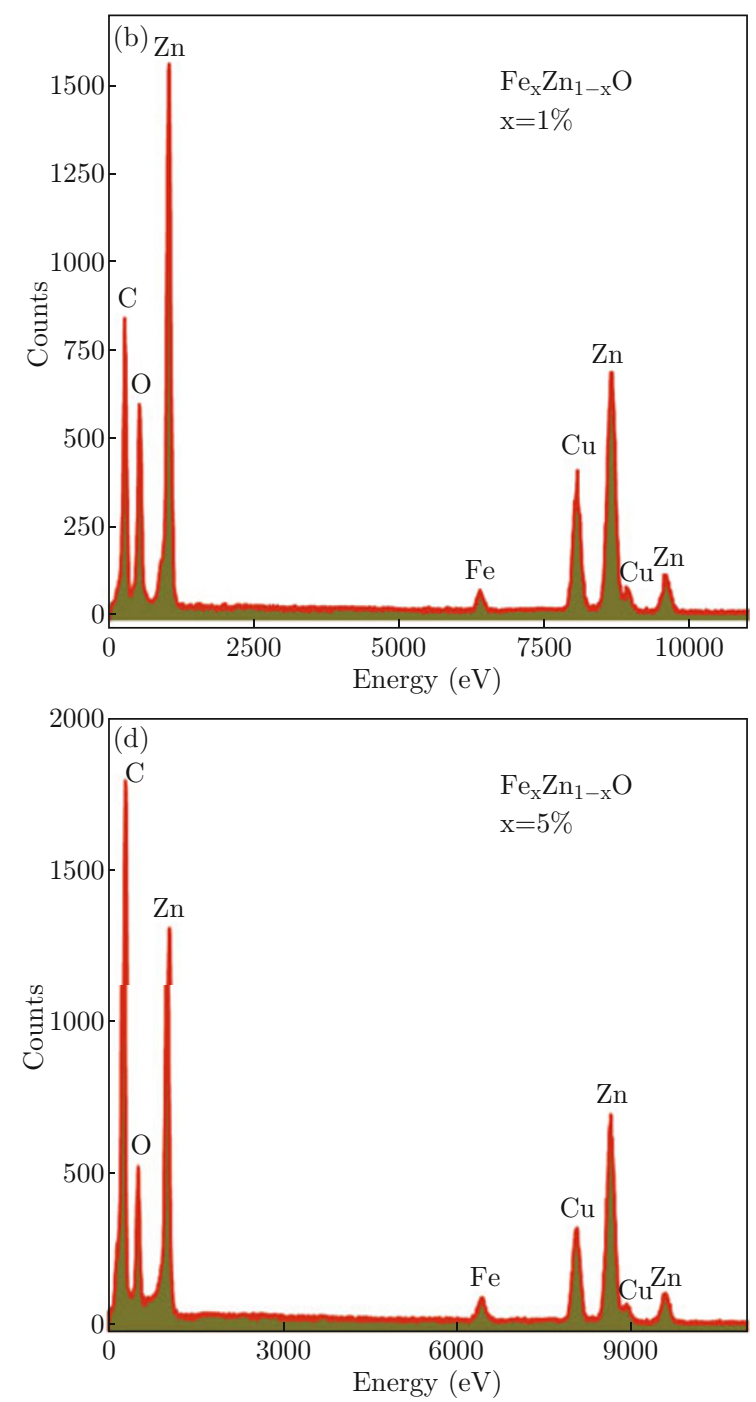

Fig. 2 EDS spectra of Fe doped ZnO Nanostructures. 
an obvious presence of Fe dopants and its variation in the concentration into nanostructures. The signals of carbon $(\mathrm{C})$ and copper $(\mathrm{Cu})$ come from the sample grid used for TEM and HRTEM investigations. The statistical analyses over dozen of individual nanowires, nanorods and nanoparticles show that $\mathrm{Fe}$ atoms are doped uniformly in the range of $\mathrm{x}=0.5 \sim 5 \%$ into nanostructures, which are almost consistent with nominal compositions used at the time of chemical reaction during the synthesis process.

\section{Crystal structure and oxidation state}

The XRD patterns of $\mathrm{Fe}_{\mathrm{x}} \mathrm{Zn}_{1-\mathrm{x}} \mathrm{O}(\mathrm{x}=0.5 \%, 1 \%$, $2 \% \& 5 \%)$ nanostructures are shown in Fig. 3(a). All the diffraction peaks can be well matched to standard wurtzite structure of $\mathrm{ZnO}$, which confirms single phase crystallinty of the prepared nanostructures. The close view of diffraction patterns in Fig. 3(b) demonstrates two obvious changes as a function of Fe dopant concentration. Firstly, the main diffraction peaks are slightly shifted towards lower angles, which are the results of successful doping [19] due to the small variations in the lattice parameters induced by dopant concentrations. The Rietveld refinement calculations of lattice parameters are tabulated in the Table1. The linear decrease of a-axis and c-axis lattice spacing with increasing concentrations of Fe indicates that the Fe dopants substitute for $\mathrm{Zn}$ ions in the lattice, and the decreasing trend can be explained with increase of $\mathrm{Fe}$ concentration. There are many Fe $(0.64 \AA)$ ions which can replace Zn $(0.74$ $\AA$ ) at octahedral sites of $\mathrm{ZnO}$ hexagonal structure, as result there is contraction in the lattice constants. Secondly, it can also be seen in Fig. 3(b) that the width of all peaks is increased with the variation of Fe concentration, which is due to preferred growth of crystal direction and changing in the grain size as different morphologies observed in the above electron microscopic investigations.

In addition, it is interesting to note that the intensity ratio of (002) polar plane to (100) non-polar plane $\left(\mathrm{I}_{(002)} / \mathrm{I}_{(100)}\right)$ is remarkably enhanced with the morphological changes (having the following order Nanowires $<$ Nanorods \& Nanoparticles) as shown in Fig. 3(b) and given in Table 1. A high ratio of $\mathrm{I}_{(002)} / \mathrm{I}_{(100)}$ with $\mathrm{Fe}$ doping shows a large fraction of polar planes, which clearly demonstrates that the polarization plays an important role for the growth direction [22]. The absence of any extra peak further rules out the formation of secondary phases and in-corroborates well with the HRTEM and SAED results for single phase crystallinty.

XPS is a surface chemical analysis technique that can be used to study the oxidation state and the concentration of elements present in the material. XPS spectra of Fe dopant for all samples have been recorded and typical spectra for $\mathrm{x}=0.5$ and $5 \%$ are displayed in Fig. 4 (a \& b). All samples exhibit same spectrum pattern with error of $\pm 0.2 \mathrm{eV}$, in which $\mathrm{Fe} 2 \mathrm{p}_{3 / 2}$ and $\mathrm{Fe} 2 \mathrm{p}_{1 / 2}$ peaks are positioned at 724.9 and $710.5 \mathrm{eV}$, respectively. The peaks positions are different from metallic $\mathrm{Fe}$ peak (Fe

Table 1 The Rietveld refinement calculations of lattice parameters.

\begin{tabular}{|c|c|c|c|c|c|c|c|c|}
\hline \multirow{2}{*}{$x(\%)$} & \multirow{2}{*}{ Morphology } & \multicolumn{3}{|c|}{ Lattice Parameters } & \multirow{2}{*}{$\mathrm{I}_{(002)} / \mathrm{I}_{(100)}$} & \multirow{2}{*}{ Band-gap (nm) } & \multicolumn{2}{|c|}{ Magnetic Parameters } \\
\hline & & $\mathrm{a}=\mathrm{b}(\AA)$ & c $(\AA)$ & $\mathrm{V}\left(\AA^{3}\right)$ & & & $\mathrm{M}_{\mathrm{s}}(\mathrm{emu} / \mathrm{g})$ & $\mathrm{H}_{\mathrm{c}}(\mathrm{Oe})$ \\
\hline 0 & Nanowires & 3.2509 & 5.2069 & 55.03 & 0.79 & 380 & - & - \\
\hline 0.5 & Nanowires & 3.2464 & 5.2044 & 54.85 & 0.91 & 388 & 0.02 & 9 \\
\hline 1 & Nanorods & 3.2439 & 5.2028 & 54.75 & 1.09 & 392 & 0.07 & 11 \\
\hline 2 & Nanorods + Nanoparticles & 3.2420 & 5.2012 & 54.67 & 1.12 & 407 & 0.20 & 139 \\
\hline 5 & Nanoparticles & 3.2401 & 5.1985 & 54.58 & 1.02 & 443 & 1.5 & 261 \\
\hline
\end{tabular}
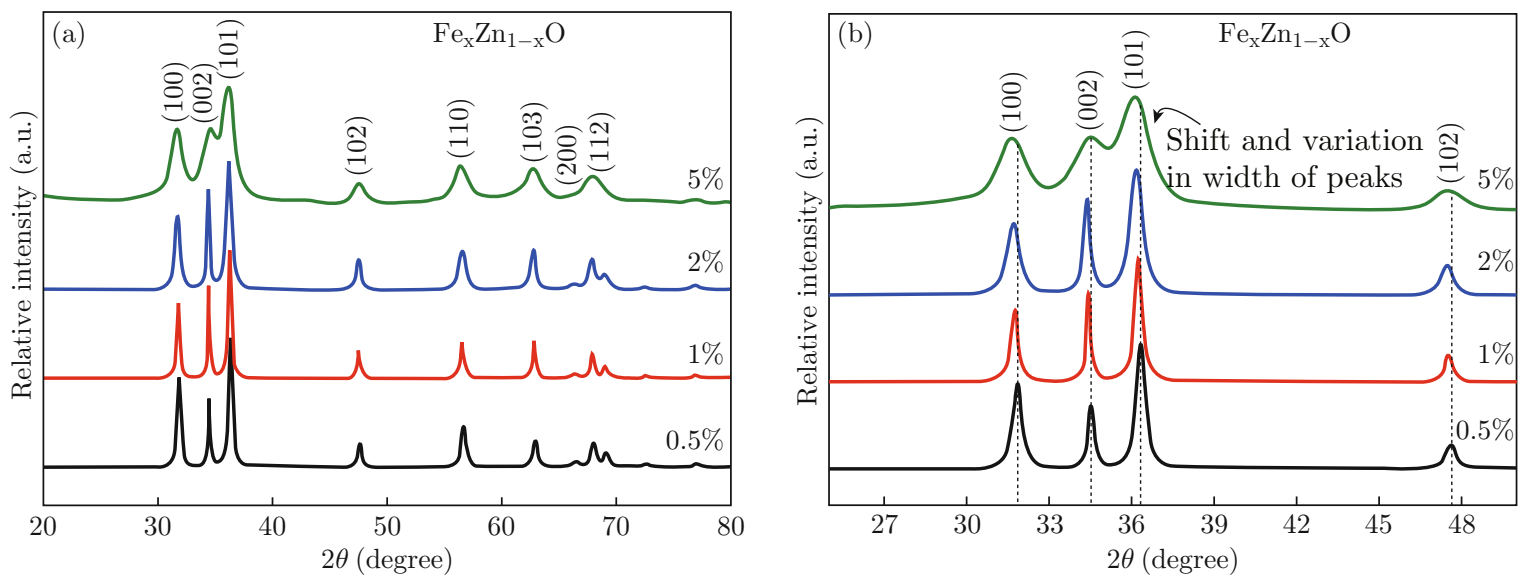

Fig. 3 (a) Full and (b) Extended XRD patterns of $\mathrm{Fe}_{\mathrm{x}} \mathrm{Zn}_{1-\mathrm{x}} \mathrm{O}$ Nanostructures. 

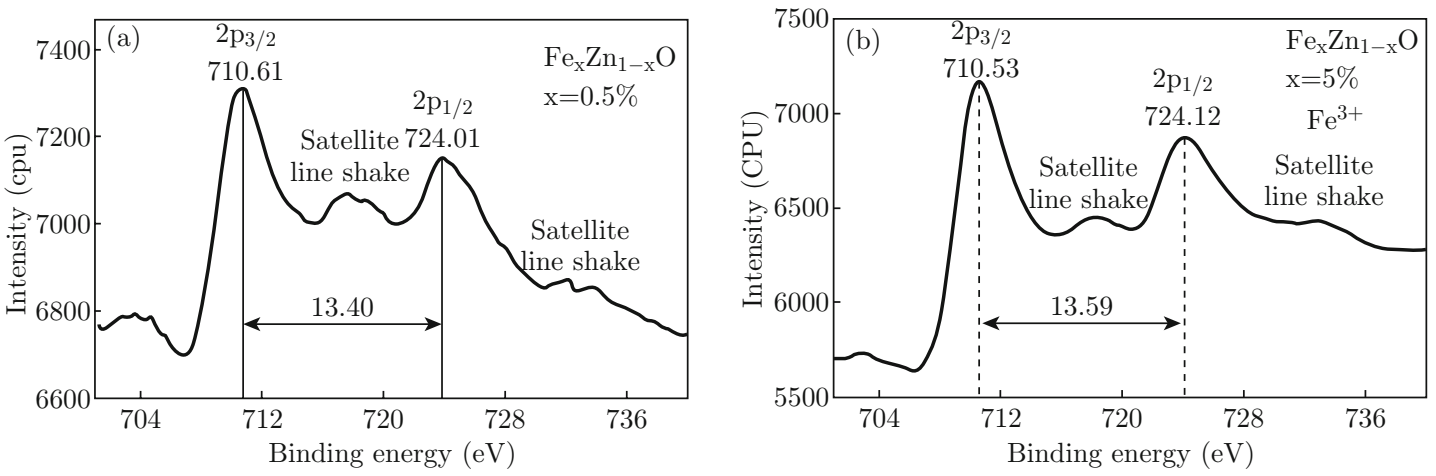

Fig. 4 Oxidation states of Fe dopants in $\mathrm{ZnO}$ Nanostructures.
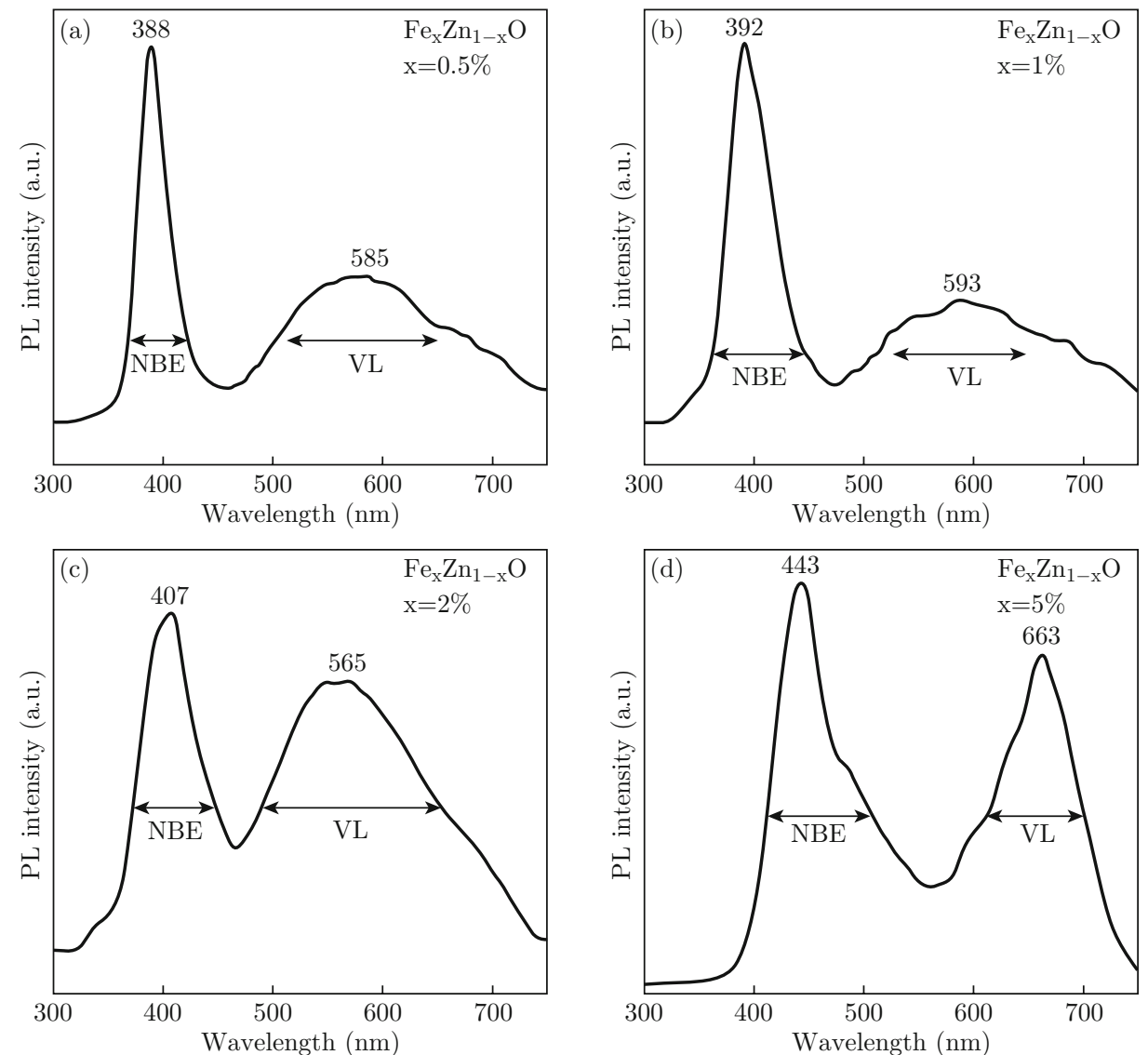

Fig. 5 Room temperature PL of Fe ( $\mathrm{x}=0.5 \%, 1 \%, 2 \%$ and $5 \%)$ doped $\mathrm{ZnO}$ Nanostructures.

$\left.2 \mathrm{p}_{1 / 2}=719.9 \mathrm{eV} \& \mathrm{Fe} 2 \mathrm{p}_{3 / 2}=706.5 \mathrm{eV}\right)$ and $\mathrm{FeO}$ $\left(\mathrm{Fe} 2 \mathrm{p}_{1 / 2}=722.3 \mathrm{eV} \& \mathrm{Fe} 2 \mathrm{p}_{3 / 2}=709.3 \mathrm{eV}\right)[23,24]$. The existence of satellite lines $\left(\mathrm{S}_{1}\right.$ and $\left.\mathrm{S}_{2}\right)$ suggests that the $\mathrm{Fe}$ is incorporated into the $\mathrm{ZnO}$ and is in $\mathrm{Fe}^{3+}$ valence state for all samples [25]. The concentration of Fe dopants in $\mathrm{ZnO}$ is in well agreement with EDS measurements and nominal compositions.

\section{Photoluminescence and ferromagnetic proper- ties}

In order to see the Fe doping induced effects on the optical properties of $\mathrm{ZnO}$, the PL spectra have been carried out and are shown in the Fig. 5. The pure $\mathrm{ZnO}$ has two emission peaks in UV $(380 \mathrm{~nm})$ and visible luminescence (VL) $(524 \mathrm{~nm})$ regions of the spectrum. The peak in UV is attributed to near band edge (NBE) transition due to interaction of exciton-exciton through collision process, while in VL usually called green emission band is assigned to the defects (such as singly ionized oxygen vacancy and $\mathrm{Zn}$ interstitial defects) $[26,27]$. The results for Fe doping demonstrate that the NBE and VL are strongly influenced from the amount of Fe doping as shown in Fig. 5 and given in Table 1. It has been interestingly found that NBE of $\mathrm{ZnO}$ shows a red shift of about 8,12, 27 and $63 \mathrm{~nm}$ 
in the band gap from that of pure $\mathrm{ZnO}$ nanowires for $0.5 \%, 1 \%, 2 \%$ and $5 \%$, respectively. The shift in the UV peak positions illustrates that the Fe doping can significantly tailor the electronic structure and bandgap of $\mathrm{ZnO}$ nanostructures. The red shift observed in the band gap of $\mathrm{Fe}$ doped $\mathrm{ZnO}$ nanoparticles is highest up to recent reported and makes this material potential for the proposed visible light (solar) photocatalyst to overcome the environmental pollution. The red-shift in band gap is usually pretended to the formation of impurity states near the bottom of conduction band of $\mathrm{ZnO}$. The merging of these states with conduction band gives rise to resultant unoccupied orbital bands [28,29]. The increase in the NBE red-shift to visible range can be understood on the basis of change in the morphology through Fe doping. The observed red-shift in the band gap may be due to strong exchange interaction present among d electrons of $\mathrm{Fe}$, and the $s$ and $p$ electrons of $\mathrm{ZnO}$ as described in the growth of nanocrystal. These strong exchange interactions among d electrons of ions and sp carriers are most typical feature of DMSs. It has been found that with the increasing Fe doping, the $s p-d$ exchange interaction becomes more dominant due to doping of more d electrons and the difference of Pauling's electronegativity of Fe (1.83) and $\mathrm{Zn}$ (1.65). The exchange interactions between electron-
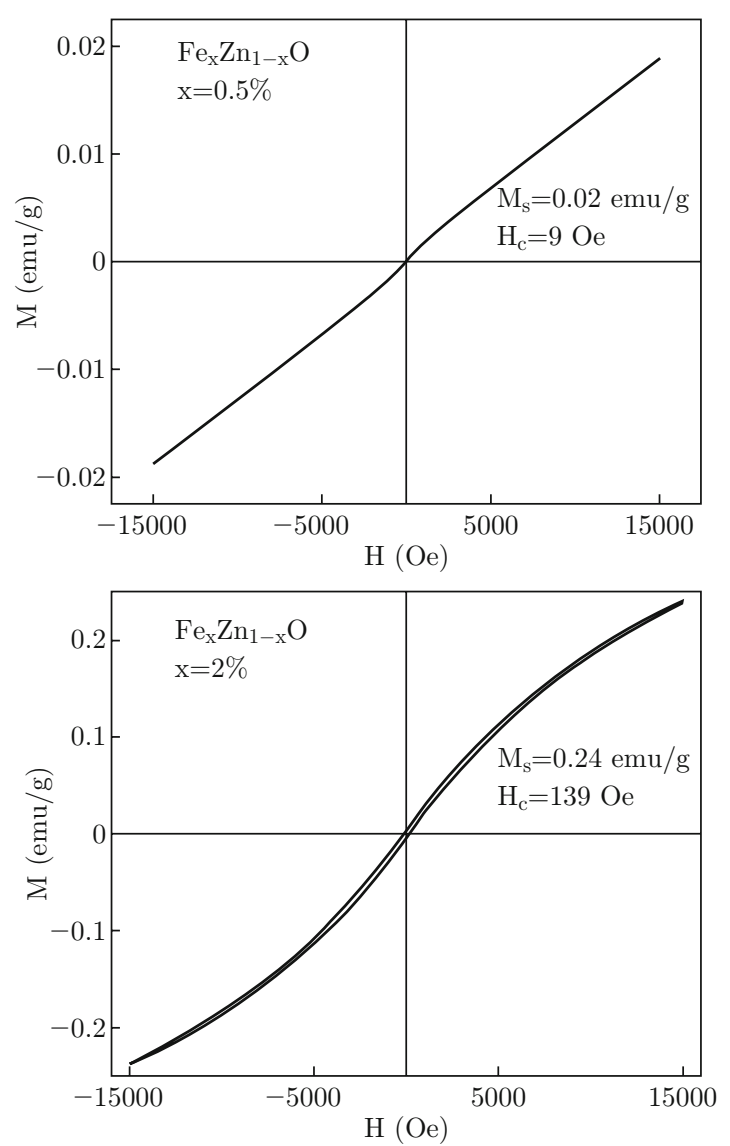

Fig. 6 Field dependent magnetization of Fe doped ZnO Nanostructures. electron and electron-impurity give rise to a negative and a positive correction to the energy of conduction and valance bands, respectively, and lead to reduce the band gap of Fe doped $\mathrm{ZnO}$ [27]. The presence of VL band for all doped samples in the PL spectra shows the existence of defects (oxygen vacancy, $\mathrm{Zn}$ interstitials and surface defects etc) in the band structure of $\mathrm{ZnO}$.

Figure 6 shows the M-H curves of $0.5 \%, 1 \%, 2 \%$ and $5 \% \mathrm{Fe}$-doped $\mathrm{ZnO}$ at room-temperature. The samples with low compositions of $0.5 \%$ and $1 \%$ exhibit both ferromagnetic and paramagnetic behaviors; while for $2 \%$ and $5 \%$ samples, there are obvious hysteresis loops. It is noteworthy that the magnetic moment $\left(M_{s}\right)$, remanence $\left(M_{r}\right)$ and coercivity $\left(H_{c}\right)$ are significantly increased with $\mathrm{Fe}$ doping concentration in $\mathrm{ZnO}$ nanostructures as shown in Fig. 6 and given in Table 1.

The exact origin of room temperature ferromagnetism (FM) in TM doped $\mathrm{ZnO}$ is not yet very clear and there are many conflicting reports about it. At this moment, the origin of the FM has usually been explained by several hypotheses: double exchange, superexchange, direct exchange, carrier mediated exchange interactions, role of anisotropy, oxygen vacancy, bound magnetic polaron overlapping and long-range exchange interactions (RKKY) etc [30-33]. In this mechanism,
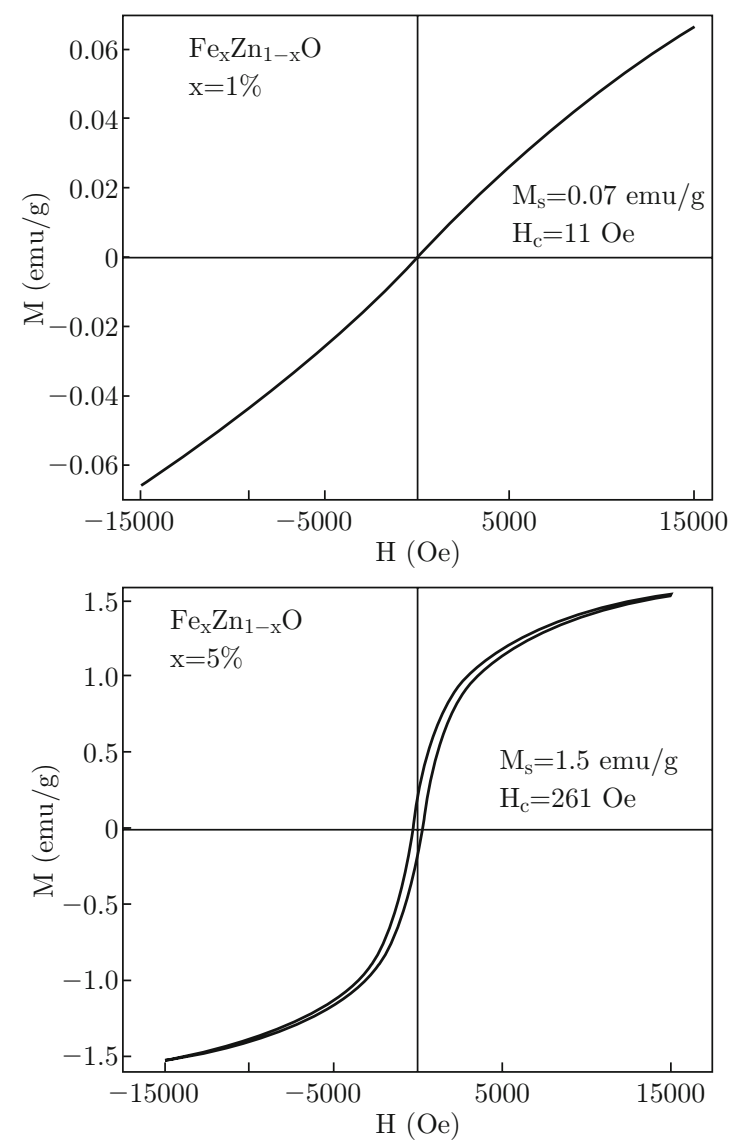

Fig. 6 Field dependent magnetization of Fe doped ZnO Nanostructures. 
role of defects (such as oxygen vacancy and zinc interstitial) is mostly considered to be an important for the observed FM in TM doped $\mathrm{ZnO}$ [34]. As it is clear from the above morphology and PL results that $s p-d$ exchange interactions and presence of defects are increased with increasing Fe concentration, which may be responsible for the origin of observed FM in Fe doped $\mathrm{ZnO}$ nanostructures. Moreover, it has been reported that grain boundaries and grain sizes also play a significant role in the ferromagnetic properties of TM doped $\mathrm{ZnO}$ [35]. The ferromagnetic behavior of Fe doped $\mathrm{ZnO}$ is believed to be more prominent, if it is comprised of nano-grains [36]. Furthermore, it has been reported that Fe doped $\mathrm{ZnO}$ samples exhibit ferromagnetic behavior only if containing certain minimum amount of grain boundaries. The amount of grain boundaries is strongly dependent on Fe concentration in the host matrix [36]. Hence, the ferromagnetic behavior of the prepared nanostructures may also be due to smaller grain sizes of the samples and presence of grain boundaries.

\section{Effect of Fe doping on $\mathrm{ZnO}$ nanostructures cy- totoxicity and ROS production}

The bio-compatibility of the prepared nanostructures has been examined on the human cell line (SH-SY5Y cells). The cells have been cultured in Dulbecco's Modification of Eagle's Medium (DMEM) with 10\% Fetal Bovine Serum (FBS). These cells have been treated with $\mathrm{ZnO}$ nanostructures doped with different Fe concentrations $(0.5,1,2,3,5 \%)$ and incubated for 24 hours at $37^{\circ} \mathrm{C}$. The effects on the cells have been studied. In order to determine the influence of the synthesized nanostructures on cell viability; CCK- 8 analysis has been performed along with phase contrast microscopy to quantify the total number of cells with and without prepared nanostructures. It is depicted in Fig. 7(a) that the prepared nanostructures have no significant effect on the viability of the tested cells. From Fig. 7(b), it is clear that $0.5 \% \mathrm{Fe}$ doped $\mathrm{ZnO}$ nanostructures has no effect on the SH-SY5Y cell viability. With the higher concentration of Fe dopant, the viability of SH-SY5Y cells is slightly affected by $\mathrm{ZnO}$ nanostructures, but all the prepared nanostructures may be thought as non toxic towards SH-SY5Y due to very low proportion of damage.

The toxicity of the prepared nanostructures has been further examined by reactive oxygen species (ROS) generation analysis. ROS generation is considered as the main mechanism through which nanomaterials cause damage to the healthy cells. It is generated on the surfaces of the nanomaterials because of the electronic properties of the material. Furthermore, ROS can be generated by the obstructed electronic transport in the
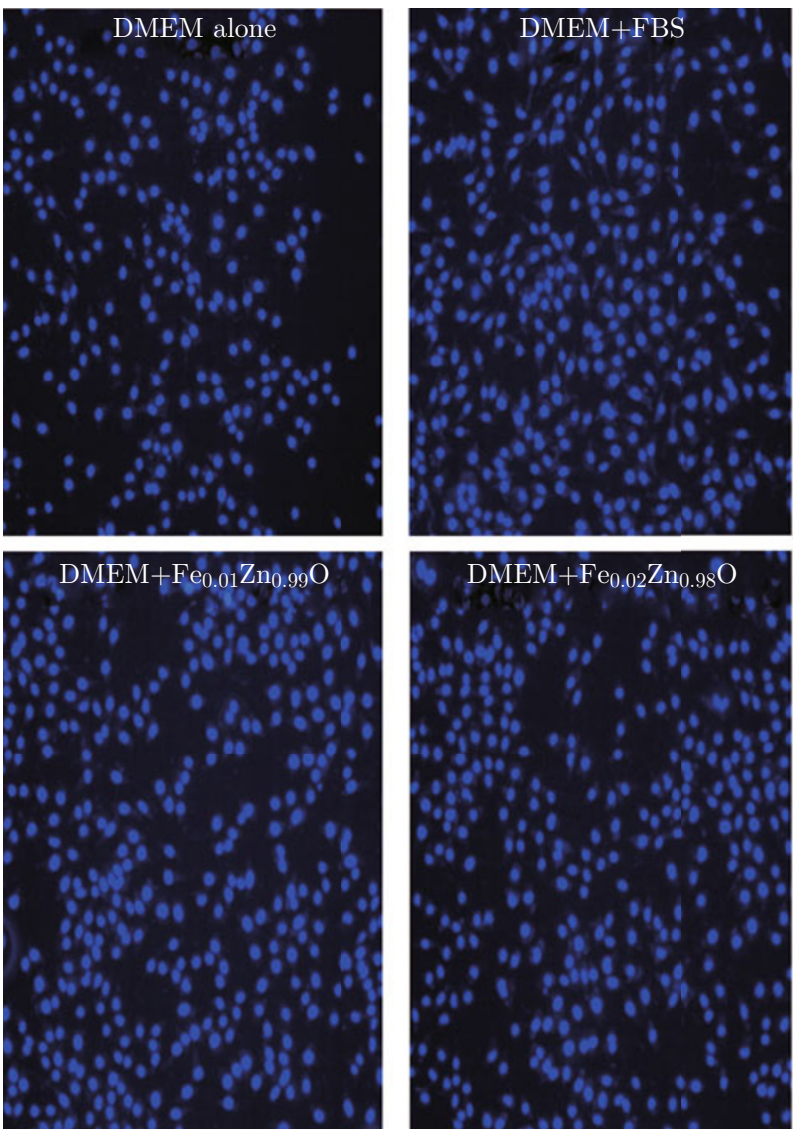

(a)

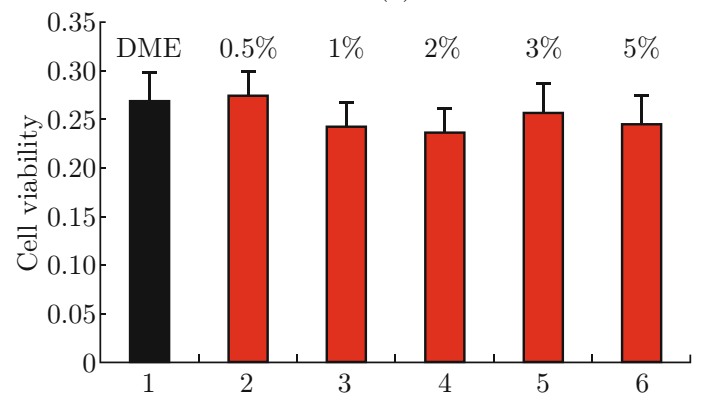

(b)

Fig. 7 (a) Florescence Microscope images of untreated and treated cell cultures. (b) Cell viability test as function of $\mathrm{ZnO}$ nanostructures doped with various concentrations of Fe.

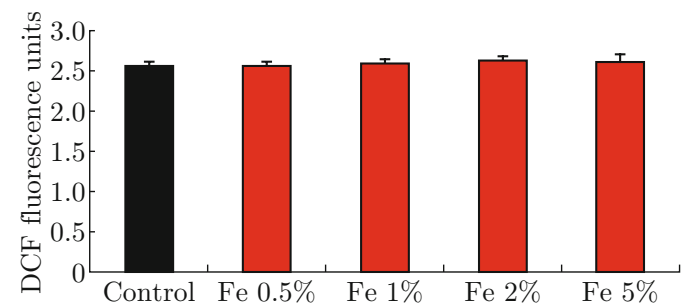

Fig. 8 Effect of Fe doped $\mathrm{ZnO}$ nanostructures on the ROS generation in SH-SY5Y Cells.

mitochondrial inner membrane [37]. The elevated generation of ROS can lead to damage cell membrane and 
cell which causes cell death [37]. ROS generation has been investigated by Flow cytometry. The cell permeable DCFH-DA dye has been used to know about the oxidative stress in cells [37]. Figure 8 depicts that a negligible amount of ROS produced in SH-SY5Y cells treated with $\mathrm{ZnO}$ nanostructures doped with different Fe concentrations $(0.5,1,2$ and 5\%).

\section{Conclusions}

In conclusion, Fe is successfully doped up to $5 \%$ into $\mathrm{ZnO}$ lattice using reproducible low-temperature simple, quick and versatile synthesis approach. The investigations reveal that the doping concentration plays a very important role to tune the growth direction of nanocrystal, band gap transitions and FM of nanomaterials. The results appear interesting in the light of the proposed possibilities for the shift of $\mathrm{ZnO}$ band gap to represent an alternative of $\mathrm{TiO}_{2}$ for solar light activated photocatalyst, magneto optical and solar cells devices for the innovative technological applications. It is concluded that $\mathrm{Fe}$ doping in $\mathrm{ZnO}$ does not change very much the cell viability and ROS production. It is also revealed that $\mathrm{Fe}$ doped $\mathrm{ZnO}$ is biosafe and biocompatible material for SH-SY5Y Cells.

\section{Acknowledgment}

This work is supported by Higher Education Commission of Pakistan, National Basic Research Program of China (2010CB934602), and National Science Foundation of China (51171007 and 51271009).

\section{References}

[1] H. Zhu, J. Iqbal, H. Xu and D. Yu, "Raman and photoluminescence properties of highly $\mathrm{Cu}$ doped $\mathrm{ZnO}$ nanowires fabricated by vapor-liquid-solid process", J. Chem. Phys. 129(12), 124713 (2008). http://dx.doi. org/10.1063/1.2981050

[2] P. V. Radovanovic and D. R. Gamelin, "Hightemperature ferromagnetism in $\mathrm{Ni}_{2}$-doped $\mathrm{ZnO}$ aggregates prepared from colloidal diluted magnetic semiconductor quantum dots", Phys. Rev. Lett. 91(15), 157202 (2003). http://dx.doi.org/10.1103/ PhysRevLett.91.157202

[3] Z. L. Wang, "Zinc oxide nanostructures: growth, properties and applications", J. Phys: Condens. Matter. 16, 829-858 (2004). http://dx.doi.org/10.1088/ 0953-8984/16/25/R01

[4] H. T. Ng, J. Han, T. Yamada, P. Nguyen, Y. P. Chen and M. Meyyappan, "Single crystal nanowire vertical surround-gate field-effect transistor", Nano Lett. 4(7), 1247-1252 (2004). http://dx.doi.org/10.1021/ nl049461z
[5] C. Soci, A. Zhang, B. Xiang, S. A. Dayeh, D. P. R. Aplin, J. Park, X. Y. Bao, Y. H. Lo and D. Wang, "ZnO nanowire UV photodetectors with high internal gain", Nano Lett. 7(4), 1003-1009 (2007). http://dx. doi.org/10.1021/n1070111x

[6] Q. H. Li, Y. X. Liang, Q. Wan and T. H. Wang, "Oxygen sensing characteristics of individual $\mathrm{ZnO}$ nanowire transistors", Appl. Phys. Lett. 85(26), 63896391 (2004). http://dx.doi.org/10.1063/1.1840116

[7] C. J. Lee, T. J. Lee, S. C. Lyu, Y. Zhang, H. Ruh and H. J. Lee, "Field emission from well-aligned zinc oxide nanowires grown at low temperature", Appl. Phys. Lett. 81(19), 3648-3650 (2002). http://dx.doi.org/ 10.1063/1.1518810

[8] M. H. Huang, S. Mao, H. Feick, H. Yan, Y. Wu, H. Kind, E. Weber, R. Russo and P. Yang, "Roomtemperature ultraviolet nanowire nanolasers", Science 292(5523), 1897-1899 (2001). http://dx.doi.org/10. 1126/science. 1060367

[9] X. D. Wang, J. H. Song, J. Liu and Z. L. Wang, "Direct-current nanogenerator driven by ultrasonic waves", Science 316(5821), 102-105 (2007). http:// dx.doi.org/10.1126/science. 1139366

[10] B. Dindar and S. Icli, "Unusual photoreactivity of zinc oxide irradiated by concentrated sunlight", J. Photochem. Photobiol. A: Chem. 140(3), 263-268 (2001). http://dx.doi.org/10.1016/ S1010-6030(01) 00414-2

[11] M. A. Behnajady, N. Modirshahla and R. Hamzavi, "Kinetic study on photocatalytic degradation of CI Acid Yellow 23 by $\mathrm{ZnO}$ photocatalyst", J. Hazard. Mater. 133(1), 226-232 (2006). http://dx.doi.org/ 10.1016/j. jhazmat. 2005.10.022

[12] S. V. Bhat and F. L. Deepak, "Tuning the bandgap of $\mathrm{ZnO}$ by substitution with $\mathrm{Mn}^{2+}, \mathrm{Co}^{2+}$ and $\mathrm{Ni}^{2+}$, Solid State Comm. 135(6), 345-347 (2005). http://dx.doi. org/10.1016/j.ssc. 2005.05.051

[13] T. Dietl, H. Ohno, F. Matsukura, J. Cibert and D. Ferrand, "Zener model description of ferromagnetism in Zinc-Blende magnetic semiconductors", Science 287(5455), 1019-1022 (2000). http://dx.doi. org/10.1126/science.287.5455.1019

[14] Quarta, R. Di Corato, L. Manna, S. Argentiere, R. Cingolani, G. Barbarella and T. Pellegrino, "Multifunctional nanostructures based on inorganic nanoparticles and oligothiophenes and their exploitation for cellular studies", J. Am. Chem. Soc. 130(32), 10545-10555 (2008). http://dx.doi.org/10. 1021/ja800102v

[15] Morfesis, D. Fairhurst, NSTI Nanotechnology Conference and Trade Show. NSTI Nanotech Anaheim, CA May 8-12, (2005).

[16] W. J. Rasmussen, E. Martinez, P. Louka, and D. G. Wingett, "Zinc oxide nanoparticles for selective destruction of tumor cells and potential for drug delivery applications", Expert Opin. Drug Deliv. 7(9), 1063-1077, (2010). http://dx.doi.org/10. $1517 / 17425247.2010 .502560$ 
[17] Rita John and Rajaram Rajakumari, "Synthesis and characterization of rare earth ion doped nano $\mathrm{ZnO",}$ Nano Micro Lett. 4(2), 65-72 (2012). http://dx.doi. org/10.3786/nml.v4i2.p65-72

[18] J. Iqbal, B. Wang, X. F. Liu, H. Be, D. P. Yu and R. H. Yu, "Oxygen-vacancy-induced green emission and room-temperature ferromagnetism in Ni-doped ZnO nanorods", New Journal Phys. 11(6), 063009 (2009). http://dx.doi.org/10.1088/1367-2630/11/ $6 / 063009$

[19] Y. J. Li, C. Y. Wang, M. Y. Lu, K. M. Li and L. J. Chen, "Electrodeposited hexagonal ringlike superstructures composed of hexagonal Codoped $\mathrm{ZnO}$ nanorods with optical tuning and hightemperature ferromagnetic properties", Cryst. Growth Des. 8(8), 2598-2602 (2008). http://dx.doi.org/10. $1021 /$ cg7007864

[20] X. Y. Kong, Y. Ding, R. Yang and Z. L. Wang, "Singlecrystal nanorings formed by epitaxial self-coiling of polar nanobelts", Science 303(5662), 1348-1351 (2004). http://dx.doi.org/10.1126/science.1092356

[21] Z. Y. Tang, N. A. Kotov and M. Giersig, "Spontaneous organization of single CdTe nanoparticles into luminescent nanowires", Science 297(5579), 237-240 (2002). http://dx.doi.org/10.1126/science.1072086

[22] J. M. D. Coey, M. Viret and S. von Molnar, "Mixedvalence manganite", Adv. Phys. 48(2), 167-293 (1999). http://dx.doi.org/10.1080/000187399243455

[23] G.R. Li, T. Hu, G. L. Pan, T. Y. Yan, X. P. Gao and H. Y. Zhu, "Morphology- function relationship of $\mathrm{ZnO}$ : polar planes, oxygen vacancies, and activity", J. Phys. Chem. C 112(31), 11859-11864 (2008). http:// dx.doi.org/10.1021/jp8038626

[24] S. Baek, J. Song and S. Lim, "Improvement of the optical properties of $\mathrm{ZnO}$ nanorods by Fe doping", Physica B 399(2), 101-104 (2007). http://dx.doi.org/10. $1016 / j \cdot$ physb. 2007.05.030

[25] J. F. Moulder, William F. Stickle, Peter E. Sobol and Kenneth D. Bomben "Handbook of X-ray photoelectron spectroscopy", Perkin Elmer Corporation, 84-85, (1992).

[26] Y. W. Chen, Y. C. Liu, S. X. Lu, C. S. Xu, C. L. Shao, C. Wang, J. Y. Zhang, Y. M. Lu, D. Z. Shen and X. W. Fan, "Optical properties of $\mathrm{ZnO}$ and $\mathrm{ZnO}$ : in nanorods assembled by sol-gel method", J. Chem. Phys. 123(13), 134701 (2005). http://dx.doi.org/ 10.1063/1.2009731

[27] A. B. Djurisic, W. C. H. Choy, V. A. L. Roy, Y. H. Leung, C. Y. Kwong, K. W. Cheah, T. K. Gundu Rao, W. K. Chan, H. Fei Lui and C. Surya, "Photoluminescence and electron paramagnetic resonance of ZnO tetrapod structures", Adv. Funct. Mater. 14(9),
856-864, (2004). http://dx.doi.org/10.1002/adfm. 200305082

[28] W. Li, Y. Wang, H. Lin, S. Ismat Shah, C. P. Huang, D. J. Doren, S. A. Rykov, J. G .Chen and M. A. Barteau, "Band gap tailoring of $\mathrm{Nd}^{3+}$-doped $\mathrm{TiO}_{2}$ nanoparticles", Appl. Phys. Lett. 83(20), 4143-4145 (2003). http://dx.doi.org/10.1063/1.1627962

[29] J. Iqbal, X. Liu and H. Zhu, "Trapping of Ce electrons in band gap and room temperature ferromagnetism of $\mathrm{Ce}^{4+}$ doped $\mathrm{ZnO}$ nanowires", J. Appl. Phys. 106(8), 083515-083515-6 (2009). http://dx.doi.org/ 10.1063/1.3245325

[30] S. J. Pearton, W. H. Heo, M. Ivill, D. P. Norton and T. Steiner, "Dilute magnetic semiconducting oxides", Semicond. Sci. Technol. 19(10), 54-59 (2004). http:// dx.doi.org/10.1088/0268-1242/19/10/R01

[31] M. Venkatesan, C. B. Fitzgerald, J. G. Lunney and J. M. D. Coey, "Anisotropic ferromagnetism in substituted zinc oxide", Phys. Rev. Lett. 93(17), 177206 (2004). http://dx.doi.org/10.1103/ PhysRevLett.93.177206

[32] M. Venkatesan, C. B. Fitzgerald and J. M. D. Coey, "Thin films: unexpected magnetism in a dielectric oxide", Nature 430(7000), 630-630 (2004). http://dx. doi.org/10.1038/430630a

[33] N. Khare, M. J. Kappers, M.Wei, M.G. Blamire and J. L. Macmanus-Driscoll, "Defect-induced ferromagnetism in Co-doped ZnO", Adv. Mater. 18(11), 1449-1452 (2006). http://dx.doi.org/10. 1002/adma. 200502200

[34] B. B. Straumal, S. G. Protasova, d, A. A. Mazilkin, G. Schütz, E. Goering, B. Baretzky and P. B. Straumal, "Ferromagnetism of zinc oxide nanograined films", JETP Letters. 97(6), 367-377 (2013). http://dx . doi . org/10.1134/S0021364013060143

[35] B. B. Straumal, S. G. Protasova, A. A. Mazilkin, T. Tietze, E. Goering, G. Schütz, P. B. Straumal and B. Baretzky, "Ferromagnetic behaviour of Fe-doped $\mathrm{ZnO}$ nanograined films", Beilstein J. Nanotechnol. 4(1), 361-369 (2013). http://dx.doi.org/10.3762/ bjnano. 4.42

[36] T. Xia, M. Kovochich, M. Liong, L. Madler, B. Gilbert, H. Shi, J. I. Yeh, J. I. Zink and A. E. Nel, "Comparison of the mechanism of toxicity of zinc oxide and cerium oxide nanoparticles based on dissolution and oxidative stress properties", ACS Nano. 2(10), 2121-2134 (2008). http://dx.doi.org/10.1021/nn800511k

[37] C. Hanley, A. Thurber, C. Hanna, A. Punnoose. J. Zhang and D. G. Wingett, "The influences of cell type and $\mathrm{ZnO}$ nanoparticle size on immune cell cytotoxicity and cytokine induction", Nanoscale Res. Lett. 4(12), 1409-1420 (2009). http://dx.doi.org/ $10.1007 /$ s $11671-009-9413-8$ 